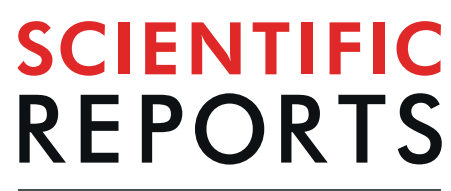

natureresearch

\title{
Synthesis of new isoquinoline- base-oxadiazole derivatives as potent inhibitors of thymidine phosphorylase and molecular docking study
}

\begin{abstract}
Khalid Zaman ${ }^{1}$, Fazal Rahim ${ }^{1}$, Muhammad Taha ${ }^{2 *}$, Abdul Wadood ${ }^{3}$, Syed Adnan Ali Shah ${ }^{4,5}$, Qamar Uddin Ahmed ${ }^{6}$ \& Zainul Amiruddin Zakaria7,8*

Here in this study regarding the over expression of TP, which causes some physical, mental and socio problems like psoriasis, chronic inflammatory disease, tumor angiogenesis and rheumatoid arthritis etc. By this consideration, the inhibition of this enzyme is vital to secure life from serious threats. In connection with this, we have synthesized twenty derivatives of isoquinoline bearing oxadiazole (1-20), characterized through different spectroscopic techniques such as HREI-MS, ${ }^{1} \mathrm{H}-\mathrm{NMR}$ and ${ }^{13} \mathrm{C}-\mathrm{NMR}$ and evaluated for thymidine phosphorylase inhibition. All analogues showed outstanding inhibitory potential ranging in between $1.10 \pm 0.05$ to $54.60 \pm 1.50 \mu \mathrm{M}$. 7-Deazaxanthine $\left(\mathrm{IC}_{50}=38.68 \pm 1.12 \mu \mathrm{M}\right)$ was used as a positive control. Through limited structure activity relationships study, it has been observed that the difference in inhibitory activities of screened analogs are mainly affected by different substitutions on phenyl ring. The effective binding interactions of the most active analogs were confirmed through docking study.
\end{abstract}

Cancer is considered as the second foremost cause of mortality world widely, therefore cancer therapy has gained considerable attention. Cancer therapy through target-based drug has developed many rationally designed inhibitors of thymidylate synthase ${ }^{1-3}$, glycinamide ribonucleotide formyltransferase ${ }^{4}$, and purine nucleoside phosphorylase $^{5}$, that can impede the acute biochemical pathway or completely stop the DNA replication and subsequently prevent the growth of cancer cells. Ongoing research in this arena has identified TP as restorative target for cancer therapy. Thymidine phosphorylase is found in both prokaryotic and eukaryotic domains that accelerates the changeover of thymidine into thymine and $2^{\prime}$-deoxy-D-ribose 1-phosphates through reverse phosphoryl$\operatorname{sis}^{6-8}$. Furthermore, dephosphorylation of 2'-deoxy-D-ribose 1-phosphates produces 2'-deoxy-D-ribose, which prompts the secretion of vascular endothelial growth factor (VEGF). Vascular endothelial growth factor favors a series of process like secretion of matrix metalloprotieneses, migration and proliferation of endothelial cells to tumor tissue, endorses cancer metastasis and instant generation of new blood vessels ${ }^{9}$. Moreover, high level of thymidine phosphorylase favors some cancerous problems such as breast tumor, colorectal, pancreatic, ovarian and hypoproiferative disease $\mathrm{e}^{10-13}$.

\footnotetext{
${ }^{1}$ Department of Chemistry, Hazara University, Mansehra, 21300, Khyber Pakhtunkhwa, Pakistan. ${ }^{2}$ Department of Clinical Pharmacy, Institute for Research and Medical Consultations (IRMC), Imam Abdulrahman Bin Faisal University, P. O. Box 1982, Dammam, 31441, Saudi Arabia. ${ }^{3}$ Department of Biochemistry, Abdul Wali Khan University Mardan, Mardan, 23200, Pakistan. ${ }^{4}$ Faculty of Pharmacy, Universiti Teknologi MARA Cawangan Selangor Kampus Puncak Alam, 42300 Bandar Puncak Alam, Selangor, D.E., Malaysia. ${ }^{5}$ Atta-ur-Rahman Institute for Natural Products Discovery (AuRIns), Universiti Teknologi MARA Cawangan Selangor Kampus Puncak Alam, 42300 Bandar Puncak Alam, Selangor, D.E., Malaysia. ${ }^{6}$ Department of Pharmaceutical Chemistry, Kulliyyah of Pharmacy, International Islamic University Malaysia, Pahang DM, Kuantan, 25200, Malaysia. ${ }^{7}$ Department of Biomedical Science, Faculty of Medicine and Health Sciences, Universiti Putra Malaysia, 43400, Serdang, Selangor, Malaysia. ${ }^{8}$ Halal Institute Research Institute, Universiti Putra Malaysia, 43400, Serdang, Selangor, Malaysia. *email: mtaha@iau.edu.sa; zaz@ upm.edu.my
} 
<smiles>Oc1cccc(-c2csc3nnc(-c4cnc5ccccc5n4)n23)c1O</smiles>

3-(3-(quinoxalin-2-yl)thiazolo[2,3-c][1,2,4]triazol-5yl)benzene-1,2-diol ${ }^{43}$<smiles>Fc1ccc(F)c(CN2CCN(C(=S)Nc3ccc(C(F)(F)F)cc3)CC2)c1</smiles>

4-(2,5-difluorobenzyl)- $N$-(4-(trifluoromethyl)phenyl)piperazine-1carbothioamide ${ }^{44}$<smiles>O=C1C=CC2C=C(C(=O)N/N=C\C3=C(Cl)C4C=CC=CC4OC3=O)C=CC2N1</smiles>

Figure 1. Reported lead candidates.

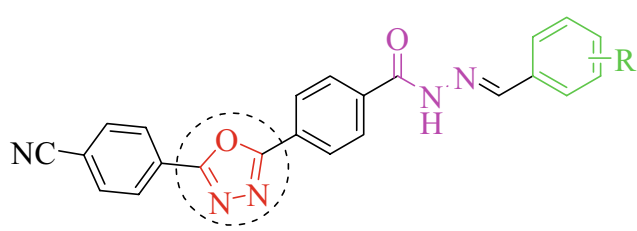

$(E)-N$ '-substituted-benzylidene-4-(5-(4-cyanophenyl)-1,3,4oxadiazol-2-yl)benzohydrazide

$$
\mathrm{IC}_{\mathbf{5 0}}=\mathbf{1 . 1 0} \pm 0.05 \text { to } 49.60 \pm 1.30 \mu \mathrm{M}
$$

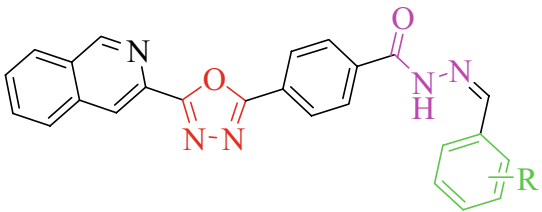

New synthetic analogues 1-20

Potent thymidine phosphorylase inhibitors

Thymidine phosphorylase inhibitors ${ }^{46}$

Figure 2. Rational study of the current work.

Thymidine phosphorylase inhibitors suppress the growth of tumor cells via disintegrating the production of $2^{\prime}$-deoxy-D-ribose ${ }^{14}$. Currently, FDA has approved Lonsurf (trifluridine/tipiracil) as TP inhibiter, but it was found with some side effects like neutropenia, anemia and myelosuppression. Therefore, it is very crucial to develop TP inhibitors with least possible side effects and have the potentials to overcome the instant generation of new blood vessels and block the growth of tumor cells. In this regard, synthetic and medicinal chemists have reported various heterocyclic analogs as TP inhibitors ${ }^{10,15-21}$.

Isoquinoline alkaloids are $\mathrm{N}$-containing heterocyle, widely distributed in nature and have manifested its broad spectrum potentials like immunoregulation, analgesic, anti-bacterial, anti-plateleted aggregation, anti-hypertensive and anti-arrhythmia ${ }^{22,23}$. Most of isoquinoline alkaloids have been confirmed for their therapeutic potentials against cancer related enzymes such as cyclin dependent kinase $4^{24}$, isosine 50-manophosphate dehydrogenase $\mathrm{e}^{25}$, mammalian sterile 20 kinase $^{26}$, topoisomerase $\mathrm{1}^{27}$ and 3 -formylcoumarin.

Our research group with continuous sincere efforts for many years have designed and synthesized various heterocyclic moieties with most promising potentials ${ }^{28-41}$. We have already reported quinoxaline, piperazine and 3 -formylcoumarin analogues (Fig. 1) as potent TP inhibiter ${ }^{42-44}$.

Comparatively with our previous work (Fig. 2$)^{45}$, here, we have synthesized new isoquinoline based oxadiazole analogs for TP inhibitor to explore further their TP inhibitory potentials in search of lead candidate to limit the effect of TP over expression. The new compounds have been synthesized based on common features of our previously reported compounds.

\section{Results and Discussion}

Chemistry. Methyl isoquinoline-3-carboxylate (I) and hydrazine hydrate were reacted in methanol, refluxed for 6 hrs to give isoquinoline-3-carbohydrazide (II) as first intermediate product. The intermediate product (II) was further reacted with methyl-4-formyl benzoate in $20 \mathrm{ml}$ ethanol, acidified the solution by few drops of acetic acid and refluxed the reaction mixture for $3 \mathrm{hrs}$ to give methyl 4-((2-(isoquinoline-3-carbonyl) hydrazono) methyl)benzoate (III) as second intermediate product. The intermediate product (III) was then cyclized in DCM in the presence of phenyliodoacetate to give methyl 4-(5-(isoquinoline-3-yl)-1,3,4-oxadiazole-2-yl) benzoate (IV) as third intermediate product. The intermediate (IV) and hydrazine hydrate was mixed, then refluxed for six hrs in absolute methanol to give isoquinolinebenzohydrazide (V) as fourth intermediate product. Finally, 


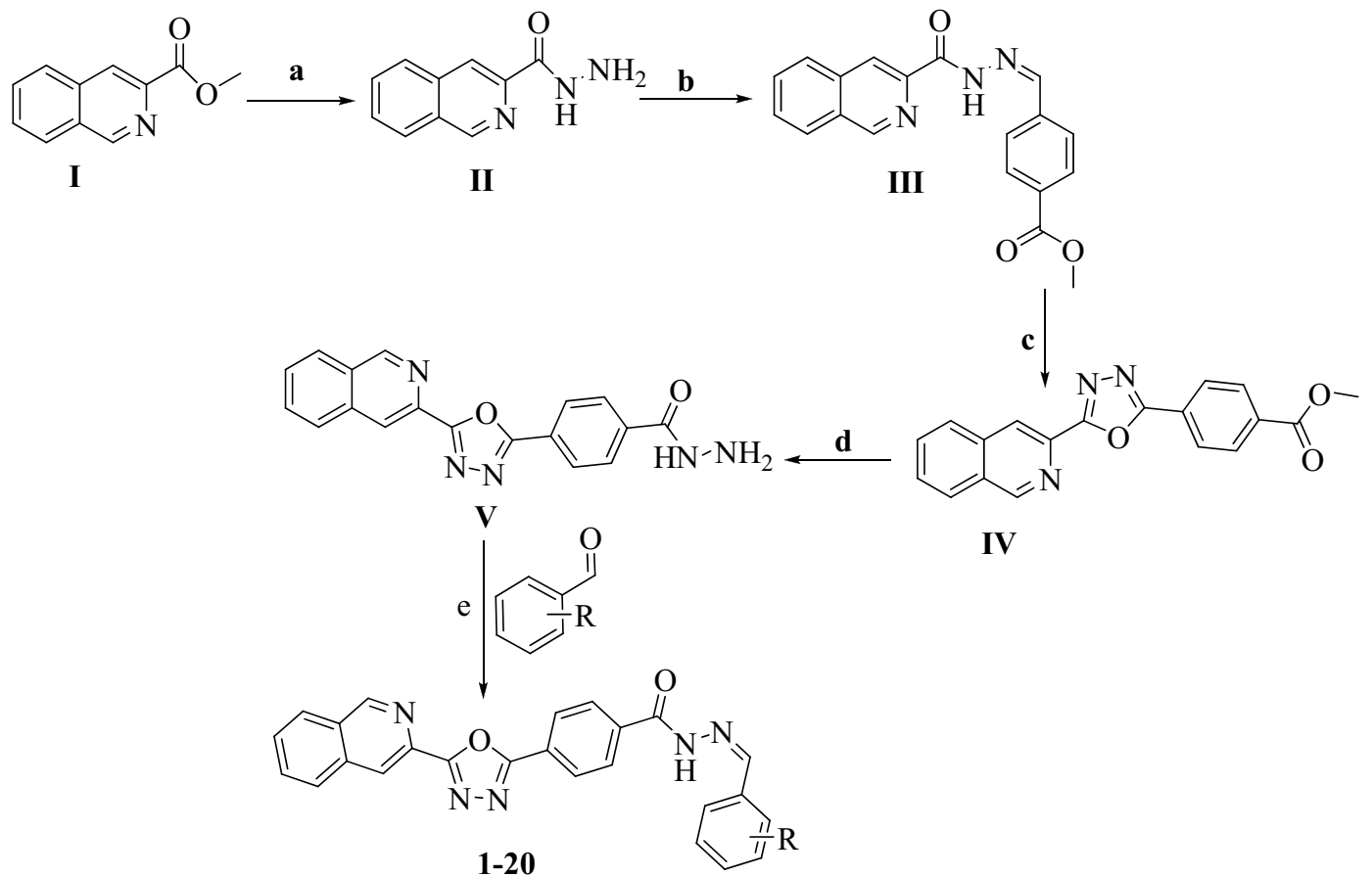

Figure 3. Reactions conditions: (a) $\mathrm{N}_{2} \mathrm{H}_{4} \cdot \mathrm{H}_{2} \mathrm{O}, \mathrm{MeOH}$, Reflux, 6h; (b) Methyl-4-formylbenzoate, $\mathrm{MeOH}$, Reflux, 6h; (c) PhI(OAc) 2 DCM, rt; (d) $\mathrm{N}_{2} \mathrm{H}_{4} \cdot \mathrm{H}_{2} \mathrm{O}, \mathrm{MeOH}$, Reflux, 6 h; (e) Substituted aromatic aldehydes/ acetophenone, $\mathrm{MeOH}, 3-4$ drops of $\mathrm{AcOH}$, Reflux, $4 \mathrm{~h}$.

the intermediate product $(\mathbf{V})$ was treated with different benzaldehyde/acetophenone in $20 \mathrm{ml}$ methanol in the presence of acetic acid and refluxed for $4 \mathrm{hrs}$ to give isoquinoline bearing oxadiazole (1-20) as final product (Fig. 3, Table 1). The final product was washed with ethanol/hexane in order to obtain pure products. All analogues (1-20) of this compound library were elucidated by spectroscopic techniques like 1H-NMR, 13C-NMR and HREI-MS.

In vitro thymidine phosphorylase activity. Twenty analogues (1-20) of isoquinoline bearing oxadiazole were synthesized and evaluated as inhibiter against thymidine phosphorylase enzyme. All analogs of the entire series were found with most potent inhibitory potential with $\mathrm{IC}_{50}$ values ranging in between $1.10 \pm 0.05 \mu \mathrm{M}$ to $54.60 \pm 1.50 \mu \mathrm{M}$ under positive control of reference drug 7-Deazaxanthine $\left(\mathrm{IC}_{50}=38.68 \pm 1.12 \mu \mathrm{M}\right)$. Seventeen analogues such as analogue 1-14 and 16-18 showed excellent inhibitory potential more better than the standard while three analogue 2,15 and 19 showed good inhibitory activity.

SAR study for thymidine phosphorylase inhibitory activity (1-20). The SAR study for particular analog among the series is mainly focused via the substitution on phenyl ring. Here in this study it was found that change of substituion or their swithing from one position to other postion on phenyl ring greatly effects the inhibitory appitude of analogues and these effects are summaraized in the below paragraphs.

If we compare analogue $15\left(\mathrm{IC}_{50}=47.30 \pm 1.20 \mu \mathrm{M}\right)$ having three methoxy groups at 2,3,4-position on phenyl ring with analogue $19\left(\mathrm{IC}_{50}=54.60 \pm 1.50 \mu \mathrm{M}\right)$ also having three methoxy groups on phenyl ring at 3,4,5-positions. Both analogues have the identical number of methoxy group on phenyl ring. The only difference found in their structures is the position of substituents on phenyl ring. With argue that substitution of methoxy groups on phenyl ring at 2,3,4-positions might activates the phenyl ring towards active of enzyme and therefore analogues 15 exhibited good activity profile than that of analogue 19 (Fig. S1).

It was seeming from the inhibitory activities that the position of substituents on phenyl ring greatly effects the inhibitory ability of the analogs. Therefore analogs 12,13 and $18\left(\mathrm{IC}_{50}=4.60 \pm 0.10 \mu \mathrm{M}, 6.00 \pm 0.20 \mu \mathrm{M}\right.$ and $7.30 \pm 0.20 \mu \mathrm{M})$ all have the same hydroxyl and methoxy groups on phenyl ring at various positions but analog 12 have hydroxyl and methoxy groups at 3, 4 positions who exhibited superior inhibitory activity then analogs 13 and 18 respectively. It clearly indicates that the position of substituents on phenyl ring might play some basic role in binding interactions of ligands and active site of enzyme (Fig. S2).

It was also shown via SAR study that some time the inhibitory activity of analog was also increased by increasing the extent of identical substituents on phenyl ring. On this regard analog $\mathbf{3}\left(\mathrm{IC}_{50}=3.30 \pm 0.10 \mu \mathrm{M}\right)$ with two chlorine atom at 2, 4 positions on phenyl ring displayed many fold better inhibitory activity then analog 6 $\left(\mathrm{IC}_{50}=16.40 \pm 0.50 \mu \mathrm{M}\right)$ bearing one more chlorine atom on phenyl ring at 4 position (Fig. S3).

Here in this study, it was clearly indicated that both electron donating group (EDG) and electron withdrawing group (EWG) on phenyl ring in all analog among the series displayed inhibitory activity. The slight difference in their potentials of analogs was mainly due to the position of substituent as well as the extent of substituent on 


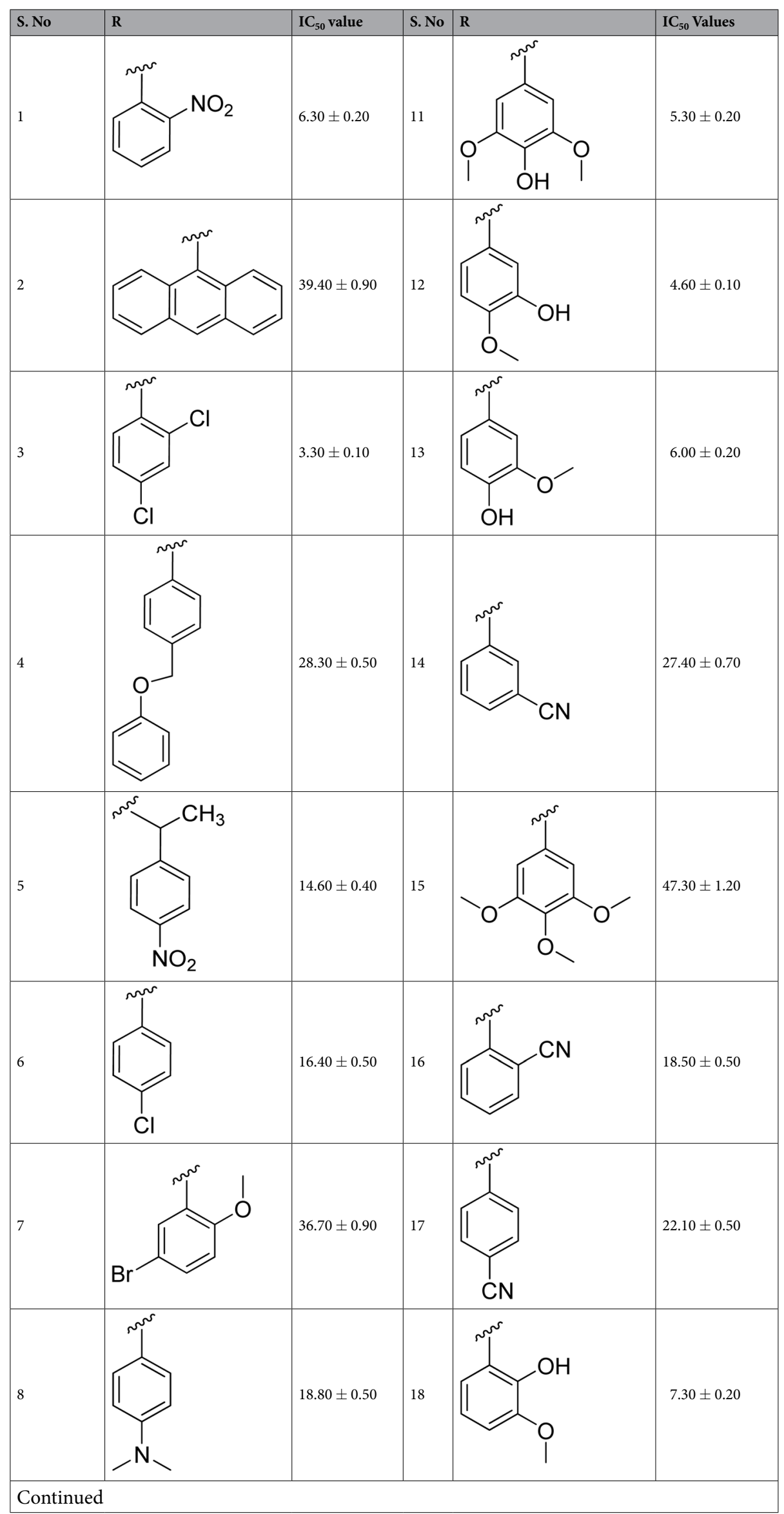




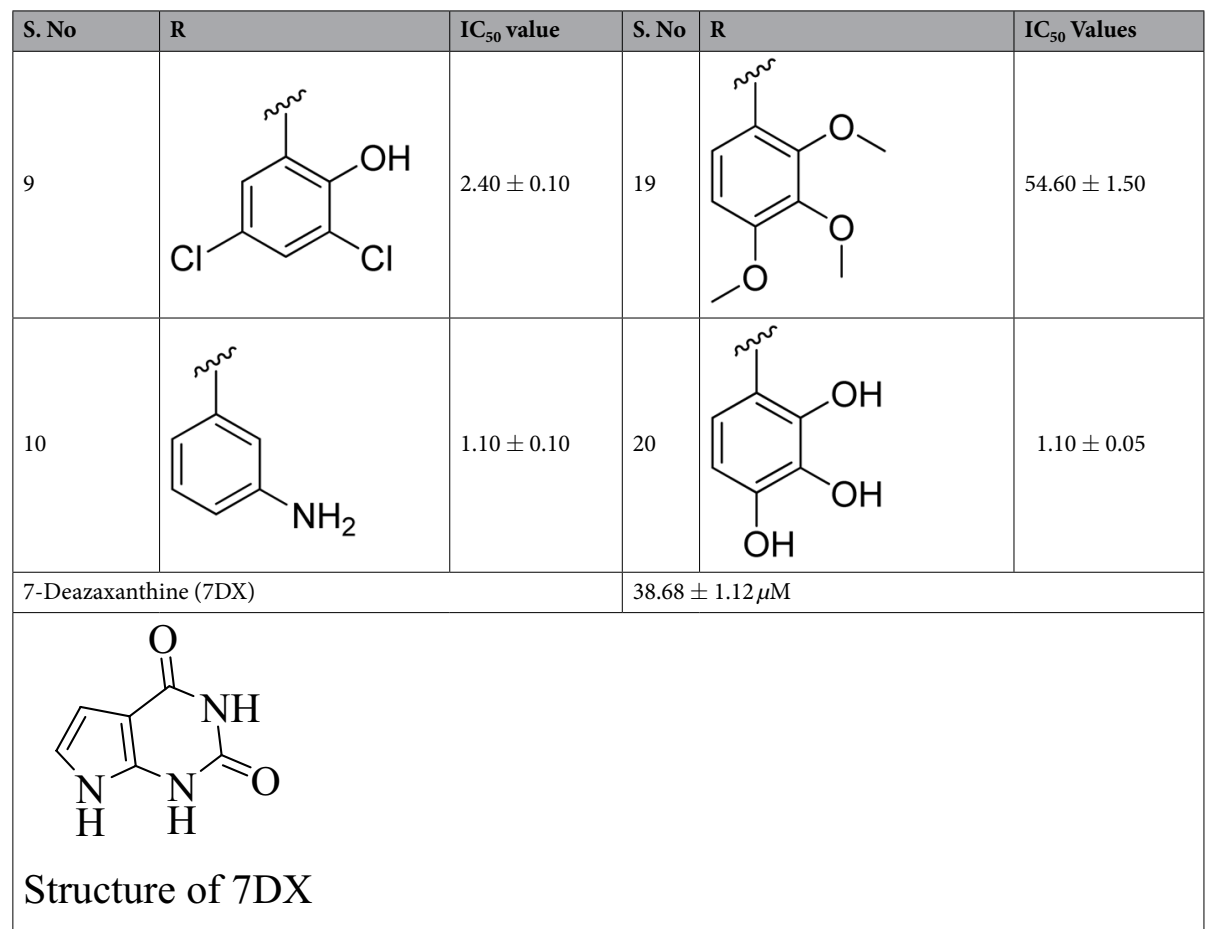

Table 1. Different notations for $\mathbf{R}$ on phenyl ring in isoquinoline bearing oxadiazole derivatives and their thymidine phosphorylase inhibitory activity. $\mathrm{IC}_{50}$ (Mean \pm Standard Deviation).
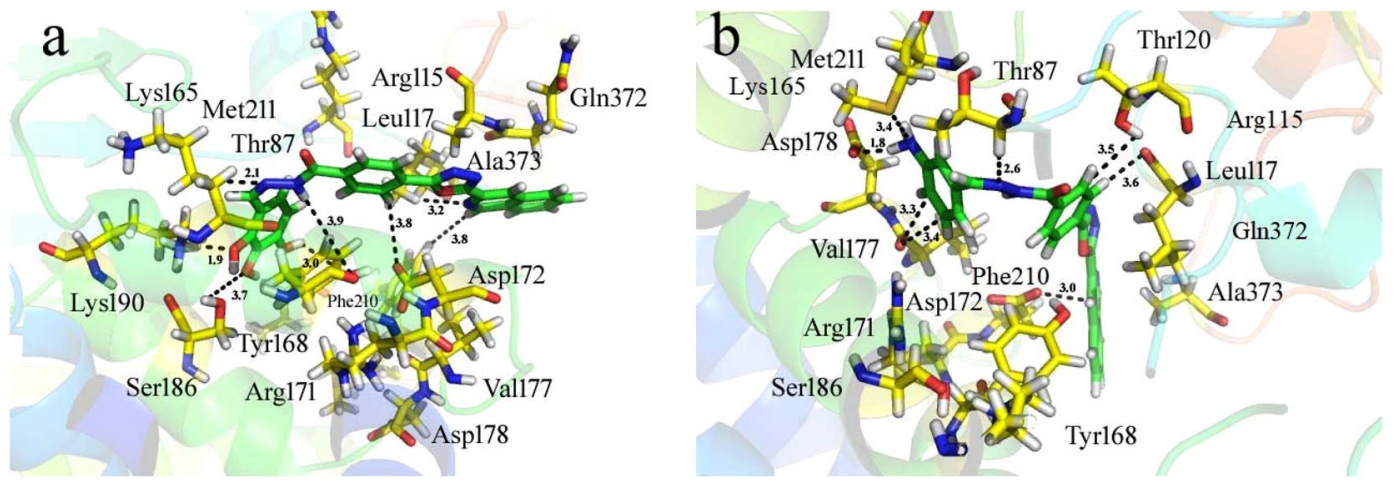

Figure 4. Docking conformations of compounds on thymidine phosphorylase enzyme. (a) 3D binding mode of compound 20 as inhibitor of thymidine phosphorylase enzyme. (b) 3D binding mode of compound 10 in binding cavity of thymidine phosphorylase enzyme. Ligands are shown green color.

phenyl ring. The binding interactions of the most active analogs and that of the active site of enzyme were confirmed through docking study.

Docking study. The concluded results of docking study of synthesized derivatives and that of thymidine phosphorylase enzyme assumed decent information about the binding pattern and have shown good correlation with experimental result. It was seeming with help of docking calculation that the top ranked conformation of all analogs approximately fitted in a good manner with active site of TP enzyme through various type of interactions i.e Thr 87, Arg 115, Leu 117, Met 211, Lys 165, Tyr 168, Arg 171, Ser 186, Asp 172, Val 177, Phe 210, Asp 178, Lys 190, Gln 372 and Ala 373 etc. The detail study about the binding interactions and docking score for almost all analogs are planned in the Table S1. The chemistry of substituents and the structural feature of most active analogs shown its efficiency in inhibitions like $\mathrm{NH}_{2}$ group have electron donating effect and certain electronegative groups i.e halogen, methoxy and $\mathrm{OH}$ groups. Among the docked analogs chlorine containing analogs were shown superior inhibition than $\mathrm{Br}$ supported by methoxy group. The interaction modes among the docked conformation of utmost analogs are demonstrated in Fig. 4(a,b). After docking, the docking conformation obtained was found with good docking score and show good result in-silico study, regarding thymidine phosphorylase enzyme inhibition. Rationally the biological evaluation and docking study of all analogs displayed good correlation result (Fig. 5). 


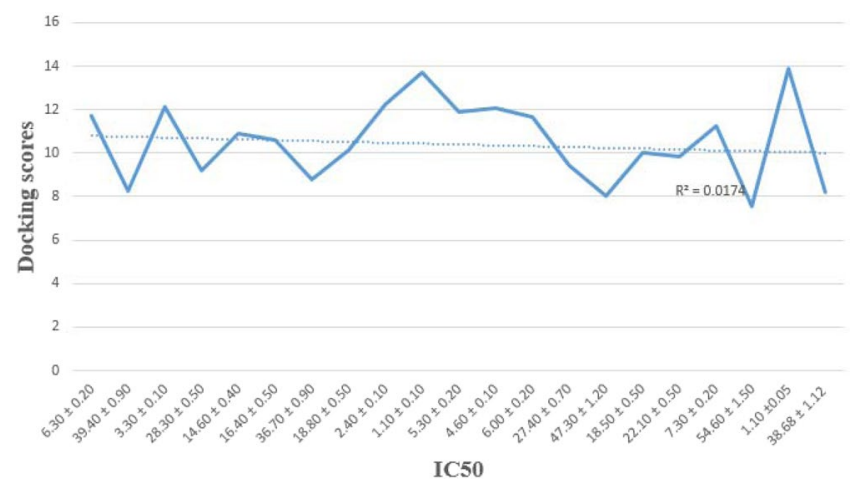

Figure 5. Predicted activity and $\mathrm{IC}_{50}$ correlation graph.

\section{Conclusion}

With variation through substituents on phenyl ring in the bulk molecule, twenty analogues (1-20) of isoquinoline bearing oxadiazole have been synthesized and screened for their thymidine phosphorylase activity. The screened result was found to be many folds better than standard drug 7-Deazaxanthine $\left(\mathrm{IC}_{50}=38.68 \pm 1.12 \mu \mathrm{M}\right)$. The structural elements in molecules were identified through docking study which play key role in the inhibitory activity. The activities of analogs were rationalized through structure-activity relationships (SAR) concluded that the whole series of analogs were comparatively identified as lead candidates which can serve for the development of new class of inhibitors.

\section{Experimental}

Material and methods. All chemicals and reagents of this protocol were purchased from sigma Aldrich, USA. All spectra's $\left(\left({ }^{1} \mathrm{H}-\mathrm{NMR},{ }^{13} \mathrm{CNMR}\right)\right.$ were performed at $500 \mathrm{MHz}$. Initially all synthetic analogues of this protocol were confirmed through thin layer chromatography on TLC plate (Kieselgel 60, 254, E. Merck, Germany) and visualized by UV lamp 254 and $365 \mathrm{~nm}$.

General procedure for the synthesis of intermediate II. Methyl isoquinoline-3-carboxylate (I) was treated with hydrazine hydrate in methanol and refluxed for 6 hrs to afford isoquinoline-3-carbohydrazide (II) as first intermediate product. Isoquinoline-3-carbohydrazide (II) formation was checked through TLC. Furthermore, to increases its purity, the intermediates was recrystallized from methanol.

General procedure for the synthesis of intermediate III. The intermediate product (II) was further reacted with methyl-4-formyl benzoate in absolute $\mathrm{MeOH}$. The reaction mixture was acidified by 3-4 drops of glacial acid and then refluxed for $3 \mathrm{hrs}$ to give methyl 4-((2-(isoquinoline-3-carbonyl)hydrazono)methyl)benzoate III as second intermediate product. The reaction mixture was stirred at room temperature till precipitate formation and filtered the crude mixture in order to obtain pure intermediate III.

General procedure for the synthesis of intermediate IV. The intermediate product (III) was then cyclized in DCM at room temperature in the presence of phenyliodoacetate to give methyl 4-(5-(isoq uinoline-3-yl)-1,3,4-oxadiazole-2-yl) benzoate IV as fourth intermediate product. Furthermore, the intermediate (IV) obtained was dissolved in $30 \mathrm{ml}$ ethyl acetate and then added $50 \mathrm{ml}$ distilled water and stirred for 35 minutes to make homogenous solution. The solution was separated layer wise in separating funnel in order to obtain pure intermediate IV.

General procedure for the synthesis of intermediate V. The pure intermediate IV was than mixed with excess of hydrazine hydrate in $\mathrm{MeOH}$ and refluxed the reaction mixture for six hrs. The product formation was checked periodically through TLC. After completion the reaction, solvent was evaporated through rota-vapor to obtain pure intermediate $\mathrm{V}$.

General procedure for the synthesis of isoquinoline-base-oxadiazole analogues (1-20). The pure intermediate $(\mathrm{V})$ was reacted with various aromatic aldehydes/acetophenone in $\mathrm{MeOH}$ and acidified the solution by 3-4 drops of glacial acetic. The reaction mixture was refluxed for 4 hrs to obtain isoquinoline-base-oxadiazole compound library (1-20). In some cases, precipitate formation was taken place within the solution which was directly filtered to obtain pure analogues. Furthermore, in case of aldehydes/acetophenone having hydroxyl/methoxy substituents, the reaction mixture was dried openly in Petridish/china-dish and then followed by workup with hexane/methanol system to obtain pure analogues.

4-(5-(isoquinolin-3-yl)-1,3,4-oxadiazol-2-yl)- $\boldsymbol{N}^{\prime}$-(2-nitrobenzylidene)benzohydrazide (1). Yield: 80\%, ${ }^{1} \mathrm{H}-\mathrm{NMR}\left(500 \mathrm{MHz}, \mathrm{DMSO}-d_{6}\right): \delta 12.0(\mathrm{~s}, 1 \mathrm{H}, \mathrm{NH}), 9.5(\mathrm{~s}, 1 \mathrm{H}, \mathrm{Ar}-\mathrm{H}), 8.9(\mathrm{~s}, 1 \mathrm{H}$, Aldehydic- H), $8.8(\mathrm{~s}, 1 \mathrm{H}, \mathrm{Ar}-\mathrm{H}), 8.3$ $(\mathrm{m}, 4 \mathrm{H}, \mathrm{Ar}-\mathrm{H}), 8.2(\mathrm{~d}, J=6.3 \mathrm{~Hz}, 1 \mathrm{H}, \mathrm{Ar}-\mathrm{H}), 8.1(\mathrm{~d}, J=6.4 \mathrm{~Hz}, 1 \mathrm{H}, \mathrm{Ar}-\mathrm{H}), 7.9(\mathrm{~d}, J=6.6 \mathrm{~Hz}, 1 \mathrm{H}, \mathrm{Ar}-\mathrm{H}), 7.8(\mathrm{t}, J=6.1 \mathrm{~Hz}$, $1 \mathrm{H}, \mathrm{Ar}-\mathrm{H}), 7.7(\mathrm{~m}, 3 \mathrm{H}, \mathrm{Ar}-\mathrm{H}), 7.6(\mathrm{t}, J=6.4 \mathrm{~Hz}, 1 \mathrm{H}, \mathrm{Ar}-\mathrm{H}) .{ }^{13} \mathrm{CNMR}\left(125 \mathrm{MHz}, \mathrm{DMSO}-d_{6}\right): \delta 164.3,164.3,163.1,152.5$, 
$150.1,147.7,143.1,136.5,134.8,133.5,132.6,131.7,130.2,130.2130 .1,130.0,129.3,128.0,127.5,127.4,127.1,126.6,125.6$, 124.0, 116.5. HREI-MS: $m / z$ calcd for $\mathrm{C}_{25} \mathrm{H}_{16} \mathrm{~N}_{6} \mathrm{O}_{4}[\mathrm{M}]+464.1233$, Found 464.1220.

$N^{\prime}$-(anthracen-9-ylmethylene)-4-(5-(isoquinolin-3-yl)-1,3,4-oxadiazol-2-yl)benzohydrazide (2). Yield: $68 \%,{ }^{1} \mathrm{H}-\mathrm{NMR}\left(500 \mathrm{MHz}, \mathrm{DMSO}-d_{6}\right): \delta 12.0(\mathrm{~s}, 1 \mathrm{H}, \mathrm{NH}), 9.5(\mathrm{~s}, 1 \mathrm{H}, \mathrm{Ar}-\mathrm{H}), 8.9$ (s, $1 \mathrm{H}$, Aldehydic$\mathrm{H}), 8.8(\mathrm{~s}, 1 \mathrm{H}, \mathrm{Ar}-\mathrm{H}), 8.3(\mathrm{~m}, 3 \mathrm{H}, \mathrm{Ar}-\mathrm{H}), 8.9$ (q, 3H, Ar-H), 7.7 (m, 7H, Ar- H), 7.4 (m, 4H, Ar-H). ${ }^{13} \mathrm{CNMR}$ $\left(125 \mathrm{MHz}\right.$, DMSO- $\left.d_{6}\right): \delta 164.3,164.3163 .1,152.5,150.1,143.2,136.5,132.5,132.3131 .6,131.7,130.3,130.1$, $130.0,129.2,128.8,128.6,128.5,128.3,128.2,128.0,127.9,127.4,127.2,127.0,126.7,125.7,125.6,125.4,125.3$, 125.0, 123.6, 116.3. HREI-MS: $m / z$ calcd for $\mathrm{C}_{33} \mathrm{H}_{21} \mathrm{~N}_{5} \mathrm{O}_{2}[\mathrm{M}]+519.1695$, Found 519.1684.

$N^{\prime}$-(2,4-dichlorobenzylidene)-4-(5-(isoquinolin-3-yl)-1,3,4-oxadiazol-2-yl)benzohydrazide (3). Yield: 78\%, ${ }^{1} \mathrm{H}-\mathrm{NMR}\left(500 \mathrm{MHz}, \mathrm{DMSO}-d_{6}\right) \delta 11.9(\mathrm{~s}, 1 \mathrm{H}, \mathrm{NH}), 9.5(\mathrm{~s}, 1 \mathrm{H}, \mathrm{Ar}-\mathrm{H}), 8.8$ (s, 1H, Aldehydic$\mathrm{H}), 8.7$ (s, $1 \mathrm{H}, \mathrm{Ar}-\mathrm{H}) 8.3(\mathrm{~m}, 3 \mathrm{H}, \mathrm{Ar}-\mathrm{H}) 8.2(\mathrm{~d}, J=6.6 \mathrm{~Hz}, 1 \mathrm{H}, \mathrm{Ar}-\mathrm{H}), 8.2(\mathrm{~m}, 2 \mathrm{H}, \mathrm{Ar}-\mathrm{H}), 8.0(\mathrm{~d}, J=7.1 \mathrm{~Hz}, 1 \mathrm{H}$, Ar- H), 7.9 (t, $J=6.3 \mathrm{~Hz}, 1 \mathrm{H}, \mathrm{Ar}-\mathrm{H}), 7.8(\mathrm{t}, J=6.1 \mathrm{~Hz}, 1 \mathrm{H}, \mathrm{Ar}-\mathrm{H}), 7.7(\mathrm{~d}, J=6.6 \mathrm{~Hz}, 1 \mathrm{H}, \mathrm{Ar}-\mathrm{H}), 7.5(\mathrm{~d}, J=6.7 \mathrm{~Hz}$, $1 \mathrm{H}, \mathrm{Ar}-\mathrm{H}) .{ }^{13} \mathrm{CNMR}\left(125 \mathrm{MHz}\right.$, DMSO- $\left.d_{6}\right): \delta 164.3,164.3,163.1,152.2,150.1,138.4,136.5,132.6,132.4,131.0$, $130.4,130.2,130.0,129.3,129.1,129.0,128.2,128.0,127.7,127.4,127.3,127.0,126.6,125.5,116.4$. HREI-MS: $\mathrm{m} / z$ calcd for $\mathrm{C}_{25} \mathrm{H}_{15} \mathrm{C}_{12} \mathrm{~N}_{5} \mathrm{O}_{2}[\mathrm{M}]+487.0603$, Found 487.0590.

4-(5-(isoquinolin-3-yl)-1,3,4-oxadiazol-2-yl)-N'-(4-(phenoxymethyl)benzylidene)benzohydrazide (4). $\quad$ Yield: $68 \%,{ }^{1} \mathrm{H}-\mathrm{NMR}\left(500 \mathrm{MHz}, \mathrm{DMSO}-d_{6}\right) \delta 12(\mathrm{~s}, 1 \mathrm{H}, \mathrm{NH}), 9.5(\mathrm{~s}, 1 \mathrm{H}, \mathrm{Ar}-\mathrm{H}), 8,8(\mathrm{~s}, 1 \mathrm{H}$, Aldehydic- H), 8.4 (s, 1H, Ar- H), 8.3 (m, 4H, Ar- H), 8.2 (d, J=6.8 Hz, 1H, Ar- H), 8.1 (m, 2H, Ar- H), 7.9 (t, $J=6.2 \mathrm{~Hz}, 1 \mathrm{H}, \operatorname{Ar}-\mathrm{H}), 7.8(\mathrm{t}, J=6.2 \mathrm{~Hz}, 1 \mathrm{H}, \operatorname{Ar}-\mathrm{H}), 7.7(\mathrm{~d}, J=6.1 \mathrm{~Hz}, 1 \mathrm{H}, \mathrm{Ar}-\mathrm{H}), 7.47(\mathrm{~m}, 2 \mathrm{H}, \mathrm{Ar}-\mathrm{H}), 7.41(\mathrm{~m}$, $4 \mathrm{H}, \mathrm{Ar}-\mathrm{H}), 7.3(\mathrm{t}, J=6.1 \mathrm{~Hz}, 1 \mathrm{H}, \mathrm{Ar}-\mathrm{H}), 4.9(\mathrm{~s}, 2 \mathrm{H}, \mathrm{OCH} 2) .{ }^{13} \mathrm{CNMR}\left(125 \mathrm{MHz}, \mathrm{DMSO}-d_{6}\right): \delta 164.3,164.3$, $163.1,158.9,152.1,150.3,146.6,138.9,136.5,132.7,132.2,130.4,130.3,130.1,129.9,129.7,129.5,129.3,129.2$, $129.1,128.9,127.8,127.8$ 127.4, 127.3, 127.0, 126.6, 125.4, 120.0, 116.6, 113.8, 113.6. HREI-MS: $\mathrm{m} / z$ calcd for $\mathrm{C}_{32} \mathrm{H}_{23} \mathrm{~N}_{5} \mathrm{O}_{3}[\mathrm{M}]+525.1801$, Found 525.1787.

4-(5-(isoquinolin-3-yl)-1,3,4-oxadiazol-2-yl)-N'-(1-(4-nitrophenyl)ethylidene)benzohydrazide (5). Yield: 68\%, ${ }^{1} \mathrm{H}-\mathrm{NMR}(500 \mathrm{MHz}, \mathrm{DMSO}-\mathrm{d} 6) \delta 11.9(\mathrm{~s}, 1 \mathrm{H}, \mathrm{NH}), 9.5$ (s, 1H, Ar- H), 8.9 (s, 1H, Ar- H), $8.3(\mathrm{~m}, 6 \mathrm{H}, \mathrm{Ar}-\mathrm{H}), 8.2(\mathrm{~m}, 3 \mathrm{H}, \mathrm{Ar}-\mathrm{H}), 7.89(\mathrm{t}, J=6 \mathrm{~Hz}, 1 \mathrm{H}, \mathrm{Ar}-\mathrm{H}), 7.87(7.8(\mathrm{t}, J=5.3 \mathrm{~Hz}, 1 \mathrm{H}, \mathrm{Ar}-\mathrm{H}), 7.7(\mathrm{~d}$, $J=6.2 \mathrm{~Hz}, 1 \mathrm{H}, \mathrm{Ar}-\mathrm{H}), 2.3$ (s, 3H, CH3). ${ }^{13} \mathrm{CNMR}(125 \mathrm{MHz}$, DMSO-d6): $\delta 164.3,164.3,163.1,152.2,150.1,150.0$, $147.5,143.3,136.4,132.5,130.6,130.3,130.1,129.5,128.3,127.9,127.7,127.5,127.3,127.2,127.1,127.0,126.7$, 125.4, 116.5, 22.6. HREI-MS: $m / z$ calcd for $\mathrm{C}_{26} \mathrm{H}_{18} \mathrm{~N}_{6} \mathrm{O}_{4}[\mathrm{M}]+478.1390$, Found 478.1374.

$N^{\prime}$-(4-chlorobenzylidene)-4-(5-(isoquinolin-3-yl)-1,3,4-oxadiazol-2-yl)benzohydrazide (6). Yield: $75 \%,{ }^{1} \mathrm{H}-\mathrm{NMR}\left(500 \mathrm{MHz}, \mathrm{DMSO}-d_{6}\right) \delta 12.1(\mathrm{~s}, 1 \mathrm{H}, \mathrm{NH}), 9.5(\mathrm{~s}, 1 \mathrm{H}, \mathrm{Ar}-\mathrm{H}), 8,8(\mathrm{~s}, 1 \mathrm{H}$, Aldehydic$\mathrm{H}), 8.7(\mathrm{~s}, 1 \mathrm{H}, \mathrm{Ar}-\mathrm{H}), 8.3(\mathrm{~m}, 4 \mathrm{H}, \mathrm{Ar}-\mathrm{H}), 8.2(\mathrm{~d}, J=6.7 \mathrm{~Hz}, 1 \mathrm{H}, \mathrm{Ar}-\mathrm{H}), 7.9(\mathrm{t}, J=6 \mathrm{~Hz}, 1 \mathrm{H}, \mathrm{Ar}-\mathrm{H}), 7.87(\mathrm{~m}, 2 \mathrm{H}$, Ar- H) $7.80(\mathrm{~m}, 2 \mathrm{H}, \mathrm{Ar}-\mathrm{H}) 7.5(\mathrm{~m}, 2 \mathrm{H}, \mathrm{Ar}-\mathrm{H}) .{ }^{13} \mathrm{CNMR}\left(125 \mathrm{MHz}\right.$, DMSO- $\left.d_{6}\right): \delta 164.3,164.3,163.1,152.2$, $150.1,146.5,136.5,136.3,132.5,131.6,130.6,130.3,130.1,130.0,129.8,129.3,128.7,128.3,128.0,127.7,127.3$, 127.1, 126.5,125.4, 116.4. HREI-MS: $\mathrm{m} / z$ calcd for $\mathrm{C}_{25} \mathrm{H}_{6} \mathrm{ClN}_{5} \mathrm{O}_{2}[\mathrm{M}]+453.0993$, Found 453.0977 .

$N^{\prime}$-(5-bromo-2-methoxybenzylidene)-4-(5-(isoquinolin-3-yl)-1,3,4-oxadiazol-2-yl)benzohydrazide (7). Yield: $75 \%,{ }^{1} \mathrm{H}-\mathrm{NMR}\left(500 \mathrm{MHz}, \mathrm{DMSO}-d_{6}\right) \delta 12.1(\mathrm{~s}, 1 \mathrm{H}, \mathrm{NH}), 9.5(\mathrm{~s}, 1 \mathrm{H}, \mathrm{Ar}-\mathrm{H}), 8.8(\mathrm{~s}, 1 \mathrm{H}$, Aldehydic-H), $8.7(\mathrm{~s}, 1 \mathrm{H}, \mathrm{Ar}-\mathrm{H}), 8.3(\mathrm{~m}, 3 \mathrm{H}, \mathrm{Ar}-\mathrm{H}), 8.1(\mathrm{~m}, 2 \mathrm{H}, \mathrm{Ar}-\mathrm{H}), 8.0(\mathrm{~d}, J=8.9 \mathrm{~Hz}, 1 \mathrm{H}, \mathrm{Ar}-\mathrm{H}), 7.9(\mathrm{t}$, $J=7.7 \mathrm{~Hz}, 1 \mathrm{H}, \mathrm{Ar}-\mathrm{H}), 7.8(\mathrm{t}, J=7.4 \mathrm{~Hz}, 1 \mathrm{H}, \mathrm{Ar}-\mathrm{H}), 7.7(\mathrm{~d}, J=6.4 \mathrm{~Hz}, 1 \mathrm{H}, \mathrm{Ar}-\mathrm{H}), 7.6(\mathrm{~d}, J=7.4 \mathrm{~Hz}, 1 \mathrm{H}, \mathrm{Ar}-\mathrm{H})$, $7.1(\mathrm{~d}, J=7.4 \mathrm{~Hz}, 1 \mathrm{H}, \mathrm{Ar}-\mathrm{H}), 3.8(\mathrm{~s}, 3 \mathrm{H}, \mathrm{OMe}){ }^{13} \mathrm{CNMR}\left(125 \mathrm{MHz}, \mathrm{DMSO}-d_{6}\right): \delta 164.3,164.3,163.1,156.1,152.2$, $150.1,146.2,136.3,134.5,132.6,131.3,130.3,130.0,129.9,129.5,128.3,127.3,127.1,127.0,126.8,125.5,118.9$, 116.6, 112.7, 110.2, 55.5. HREI-MS: $m / z$ calcd for $\mathrm{C}_{26} \mathrm{H}_{18} \mathrm{BrN}_{5} \mathrm{O}_{3}[\mathrm{M}]+527.0593$, Found 527.0582.

$N^{\prime}$-(4-(dimethylamino)benzylidene)-4-(5-(isoquinolin-3-yl)-1,3,4-oxadiazol-2-yl)benzohydrazide (8). Yield: 70\%, ${ }^{1} \mathrm{H}-\mathrm{NMR}\left(500 \mathrm{MHz}, \mathrm{DMSO}-d_{6}\right) \delta 11.9(\mathrm{~s}, 1 \mathrm{H}, \mathrm{NH}), 9.5(\mathrm{~s}, 1 \mathrm{H}, \mathrm{Ar}-\mathrm{H}), 8.8(\mathrm{~s}, 1 \mathrm{H}$, Aldehydic- H), $8.4(\mathrm{~s}, 1 \mathrm{H}, \mathrm{Ar}-\mathrm{H}), 8.3(\mathrm{~m}, 4 \mathrm{H}, \mathrm{Ar}-\mathrm{H}), 8.1(\mathrm{~d}, J=6.5 \mathrm{~Hz}, 1 \mathrm{H}, \mathrm{Ar}-\mathrm{H}), 7.9(\mathrm{t}, J=5.9 \mathrm{~Hz}, 1 \mathrm{H}, \mathrm{Ar}-\mathrm{H})$, $7.8(\mathrm{t}, J=5.9 \mathrm{~Hz}, 1 \mathrm{H}, \mathrm{Ar}-\mathrm{H}), 7.6(\mathrm{~d}, J=6.9 \mathrm{~Hz}, 1 \mathrm{H}, \mathrm{Ar}-\mathrm{H}), 7.5(\mathrm{~d}, J=6.7 \mathrm{~Hz}, 2 \mathrm{H}, \mathrm{Ar}-\mathrm{H}), 6.7(\mathrm{~d}, J=7 \mathrm{~Hz}, 2 \mathrm{H}, \mathrm{Ar}-$ $\mathrm{H}), 2.9(\mathrm{~s}, 6 \mathrm{H}, \mathrm{N}(\mathrm{CH} 3) 2) .{ }^{13} \mathrm{CNMR}\left(125 \mathrm{MHz}\right.$, DMSO- $\left.d_{6}\right): 8$ 164.3, 164.3, 163.1, 153.3, 152.2, 150.1, 146.5, 136.4, $132.5,130.5,130.3,130.1,129.3,128.5,128.3,128.0,127.4,127.2,127.1,126.7,125.7,123.1,116.6,111.9,111.8$, 41.1, 41.0. HREI-MS: $m / z$ calcd for $\mathrm{C}_{27} \mathrm{H}_{22} \mathrm{~N}_{6} \mathrm{O}_{2}[\mathrm{M}]+462.1804$, Found 462.1789 .

$N^{\prime}$-(3,5-dichloro-2-hydroxybenzylidene)-4-(5-(isoquinolin-3-yl)-1,3,4-oxadiazol-2-yl)benzohydrazide (9). Yield: $55 \%,{ }^{-1} \mathrm{H}-\mathrm{NMR}\left(500 \mathrm{MHz}\right.$, DMSO- $\left.d_{6}\right) \delta 11.9(\mathrm{~s}, 1 \mathrm{H}, \mathrm{NH}), 9.5(\mathrm{~s}, 1 \mathrm{H}, \mathrm{Ar}-\mathrm{H}), 8.8(\mathrm{~s}, 1 \mathrm{H}$, Aldehydic- H), $8.6(\mathrm{~s}, 1 \mathrm{H}, \mathrm{Ar}-\mathrm{H}), 8.3(\mathrm{~m}, 2 \mathrm{H}, \mathrm{Ar}-\mathrm{H}), 8.2(\mathrm{~m}, 3 \mathrm{H}, \mathrm{Ar}-\mathrm{H}), 7.9(\mathrm{t}, J==6.2 \mathrm{~Hz}, 1 \mathrm{H}, \mathrm{Ar}-\mathrm{H}), 7.8(\mathrm{t}$, $J=6.2 \mathrm{~Hz}, 1 \mathrm{H}, \mathrm{Ar}-\mathrm{H}), 7.7(\mathrm{~m}, 2 \mathrm{H}, \mathrm{Ar}-\mathrm{H}), 7.6(\mathrm{~d}, J=1.9 \mathrm{~Hz}, 1 \mathrm{H}, \mathrm{Ar}-\mathrm{H}) .{ }^{13} \mathrm{CNMR}\left(125 \mathrm{MHz}\right.$, DMSO- $\left.d_{6}\right): \delta 164.3$, $164.3,163.1,157.4,152.2,150.1,146.3,136.5,133.9,132.4,130.1,130.0,129.9,129.4,128.4,128.2,128.0,127.7,127.5$, 127.2,126.6, 126.4, 125.4, 121.1, 116.5. HREI-MS: $m / z$ calcd for $\mathrm{C}_{25} \mathrm{H}_{15} \mathrm{Cl}_{2} \mathrm{~N}_{5} \mathrm{O}_{3}[\mathrm{M}]+503.0552$, Found 503.0536.

$N^{\prime}$-(3-aminobenzylidene)-4-(5-(isoquinolin-3-yl)-1,3,4-oxadiazol-2-yl)benzohydrazide (10). Yield: $50 \%,{ }^{1} \mathrm{H}-\mathrm{NMR}\left(500 \mathrm{MHz}, \mathrm{DMSO}-d_{6}\right) \delta 11.9(\mathrm{~s}, 1 \mathrm{H}, \mathrm{NH}), 9.5(\mathrm{~s}, 1 \mathrm{H}, \mathrm{Ar}-\mathrm{H}), 8.8(\mathrm{~s}, 1 \mathrm{H}$, Aldehydic- H), $8.3(\mathrm{~s}, 1 \mathrm{H}, \mathrm{Ar}-$ $\mathrm{H}), 8.2(\mathrm{~m}, 4 \mathrm{H}, \mathrm{Ar}-\mathrm{H}), 8.1(\mathrm{~d}, J=6.3 \mathrm{~Hz}, 1 \mathrm{H}, \mathrm{Ar}-\mathrm{H}), 7.9(\mathrm{t}, J=5.8 \mathrm{~Hz}, 1 \mathrm{H}, \mathrm{Ar}-\mathrm{H}), 7.8(\mathrm{t}, J=5.7 \mathrm{~Hz}, 1 \mathrm{H}, \mathrm{Ar}-\mathrm{H}), 7.6(\mathrm{~d}$, $\mathrm{J}=6.8 \mathrm{~Hz}, 1 \mathrm{H}, \mathrm{Ar}-\mathrm{H}), 7.4(\mathrm{~d}, \mathrm{~J}=8.1 \mathrm{~Hz}, 1 \mathrm{H}, \mathrm{Ar}-\mathrm{H}), 7.2(\mathrm{~s}, 1 \mathrm{H}, \mathrm{Ar}-\mathrm{H}), 7.1(\mathrm{t}, J=6.7 \mathrm{~Hz}, 2 \mathrm{H}, \mathrm{Ar}-\mathrm{H}), 6.4(\mathrm{~s}, 2 \mathrm{H}, \mathrm{NH} 2)$. ${ }^{13} \mathrm{CNMR}\left(125 \mathrm{MHz}\right.$, DMSO- $\left.d_{6}\right): \delta 164.3,164.3,163.1,152.2,150.1,148.3,146.4,136.7,134.3,133.7,132.6,132.6130 .5$, 
130.3, 130.1, 129.3, 128.0, 127.5, 127.3, 126.5, 125.5, 119.1, 116.7, 117.4, 113.2. HREI-MS: $m / z$ calcd for $\mathrm{C}_{25} \mathrm{H}_{18} \mathrm{~N}_{6} \mathrm{O}_{2}$ $[\mathrm{M}]+434.1491$, Found 434.1480 .

$N^{\prime}$-(4-hydroxy-3,5-dimethoxybenzylidene)-4-(5-(isoquinolin-3-yl)-1,3,4-oxadiazol-2-yl)benzohydrazide (11). $\quad$ Yield: 65\%, ${ }^{1} \mathrm{H}-\mathrm{NMR}\left(500 \mathrm{MHz}, \mathrm{DMSO}-d_{6}\right) \delta 11.9(\mathrm{~s}, 1 \mathrm{H}, \mathrm{NH}), 9.5(\mathrm{~s}, 1 \mathrm{H}, \mathrm{Ar}-\mathrm{H}), 8.8(\mathrm{~s}, 1 \mathrm{H}$, Aldehydic-H), 8.5 (s, 1H, Ar-H), $8.3(\mathrm{~m}, 4 \mathrm{H}, \mathrm{Ar}-\mathrm{H}), 8.1(\mathrm{~d}, J=6.4 \mathrm{~Hz}, 1 \mathrm{H}, \mathrm{Ar}-\mathrm{H}), 7.9$ (t, $J=6 \mathrm{~Hz}, 1 \mathrm{H}, \mathrm{Ar}-\mathrm{H}) 7.8$ $(\mathrm{t}, J=6 \mathrm{~Hz}, 1 \mathrm{H}, \mathrm{Ar}-\mathrm{H}), 7.6(\mathrm{~d}, J=6.6 \mathrm{~Hz}, 1 \mathrm{H}, \mathrm{Ar}-\mathrm{H}), 7.2(\mathrm{~d}, J=3.5 \mathrm{~Hz}, 2 \mathrm{H}, \mathrm{Ar}-\mathrm{H}), 3.8(\mathrm{~s}, 6 \mathrm{H}, 2-\mathrm{OMe}) .{ }^{13} \mathrm{CNMR}$ $\left(125 \mathrm{MHz}, \mathrm{DMSO}-d_{6}\right): \delta 164.3,164.3,163.1,152.2,150.1,148.3,148.1,146.4,139.5,136.3,132.7,130.5,130.3$, $130.1,129.4,128.4,128.1,127.7,127.5,127.3,126.4,125.5,116.3,104.4,104.1,55.9,55.9$. HREI-MS: $\mathrm{m} / z$ calcd for $\mathrm{C}_{27} \mathrm{H}_{21} \mathrm{~N}_{5} \mathrm{O}_{5}[\mathrm{M}]+495.1543$, Found 495.1528 .

$N^{\prime}$-(3-hydroxy-4-methoxybenzylidene)-4-(5-(isoquinolin-3-yl)-1,3,4-oxadiazol-2-yl)benzohydrazide (12). Yield: 78\%, ${ }^{1} \mathrm{H}-\mathrm{NMR}\left(500 \mathrm{MHz}, \mathrm{DMSO}-d_{6}\right) \delta 11.9(\mathrm{~s}, 1 \mathrm{H}, \mathrm{NH}), 9.5(\mathrm{~s}, 1 \mathrm{H}, \mathrm{Ar}-\mathrm{H}), 9.3(\mathrm{~s}, 1 \mathrm{H}$, Aldehydic- $\mathrm{H}), 8.8(\mathrm{~s}, 1 \mathrm{H}, \mathrm{Ar}-\mathrm{H}), 8.3(\mathrm{~m}, 6 \mathrm{H}, \mathrm{Ar}-\mathrm{H}), 8.2(\mathrm{~d}, J=6.6 \mathrm{~Hz}, 1 \mathrm{H}, \mathrm{Ar}-\mathrm{H}), 7.9(\mathrm{t}, J=6.1 \mathrm{~Hz}, 1 \mathrm{H}, \mathrm{Ar}-\mathrm{H})$, $7.8(\mathrm{t}, J=6.1 \mathrm{~Hz}, 1 \mathrm{H}, \mathrm{Ar}-\mathrm{H}), 7.1(\mathrm{~d}, J=6.4 \mathrm{~Hz}, 1 \mathrm{H}, \mathrm{Ar}-\mathrm{H}), 7.1(\mathrm{~d}, J=6.7 \mathrm{~Hz}, 1 \mathrm{H}, \mathrm{Ar}-\mathrm{H}), 3.8(\mathrm{~s}, 3 \mathrm{H}, \mathrm{OMe})$. ${ }^{13}$ CNMR (125 MHz, DMSO- $\left.d_{6}\right): \delta 164.3,164.3,163.1,152.4,152.2,150.1,147.1,146.5,136.5,132.5,130.9,130.5$, $130.3,130.2,129.3,128.1,127.8,127.6,127.4,126.7,125.6,122.6,116.5,115.7,112.1,55.9$. HREI-MS: $\mathrm{m} / \mathrm{z}$ calcd for $\mathrm{C}_{26} \mathrm{H}_{19} \mathrm{~N}_{5} \mathrm{O}_{4}[\mathrm{M}]+465.1437$, Found 465.1420.

$N^{\prime}$-(4-hydroxy-3-methoxybenzylidene)-4-(5-(isoquinolin-3-yl)-1,3,4-oxadiazol-2-yl)benzohydrazide (13). Yield: 70\%, ${ }^{1} \mathrm{H}-\mathrm{NMR}\left(500 \mathrm{MHz}, \mathrm{DMSO}-d_{6}\right) \delta 11.9(\mathrm{~s}, 1 \mathrm{H}, \mathrm{NH}), 9.5(\mathrm{~s}, 1 \mathrm{H}, \mathrm{Ar}-\mathrm{H}), 8.8(\mathrm{~s}, 1 \mathrm{H}$, Aldehydic- H), $8.5(\mathrm{~s}, 1 \mathrm{H}, \mathrm{Ar}-\mathrm{H}), 8.3(\mathrm{~m}, 6 \mathrm{H}, \mathrm{Ar}-\mathrm{H}), 8.1(\mathrm{~d}, J=6.6 \mathrm{~Hz}, 1 \mathrm{H}, \mathrm{Ar}-\mathrm{H}), 7.9(\mathrm{t}, J=6.1 \mathrm{~Hz}, 1 \mathrm{H}, \mathrm{Ar}-$ $\mathrm{H}), 7.8(\mathrm{t}, J=6.1 \mathrm{~Hz}, 1 \mathrm{H}, \mathrm{Ar}-\mathrm{H}), 7.1(\mathrm{~d}, J=6.4 \mathrm{~Hz}, 1 \mathrm{H}, \mathrm{Ar}-\mathrm{H}), 6.8(\mathrm{~d}, J=6.4 \mathrm{~Hz}, 1 \mathrm{H}, \mathrm{Ar}-\mathrm{H}), 3.8(\mathrm{~s}, 3 \mathrm{H}, \mathrm{OMe})$. ${ }^{13} \mathrm{CNMR}\left(125 \mathrm{MHz}\right.$, DMSO- $\left.d_{6}\right): \delta 164.3,164.4,163.1,152.5,151.1,149.2,146.9,136.5,132.6,132.6,130.7,130.5$, $130.5,129.9,129.4,128.2,127.5,127.3,127.2,126.5,125.6,122.7,117.1,116.4,112.2,55.9$. HREI-MS: $m / z$ calcd for $\mathrm{C}_{26} \mathrm{H}_{19} \mathrm{~N}_{5} \mathrm{O}_{4}[\mathrm{M}]+465.1437$, Found 465.1420 .

$N^{\prime}$-(3-cyanobenzylidene)-4-(5-(isoquinolin-3-yl)-1,3,4-oxadiazol-2-yl)benzohydrazide (14). Yield: 65\%, ${ }^{1} \mathrm{H}-\mathrm{NMR}\left(500 \mathrm{MHz}\right.$, DMSO- $\left.d_{6}\right) \delta 11.9(\mathrm{~s}, 1 \mathrm{H}, \mathrm{NH}), 9.5(\mathrm{~s}, 1 \mathrm{H}, \mathrm{Ar}-\mathrm{H}), 8,8(\mathrm{~s}, 1 \mathrm{H}$, AldehydicH), $8.5(\mathrm{~s}, 1 \mathrm{H}, \mathrm{Ar}-\mathrm{H}), 8.3(\mathrm{~m}, 4 \mathrm{H}, \mathrm{Ar}-\mathrm{H}), 8.2(\mathrm{~m}, 4 \mathrm{H}, \mathrm{Ar}-\mathrm{H}), 8.1(\mathrm{~d}, J=6.4 \mathrm{~Hz}, 1 \mathrm{H}, \mathrm{Ar}-\mathrm{H}), 7.9(\mathrm{t}, J=6.3 \mathrm{~Hz}$, $1 \mathrm{H}, \mathrm{Ar}-\mathrm{H}), 7.8(\mathrm{t}, J=6.3 \mathrm{~Hz}, 1 \mathrm{H}, \mathrm{Ar}-\mathrm{H}) 7.1(\mathrm{~d}, J=6.2 \mathrm{~Hz}, 1 \mathrm{H}, \mathrm{Ar}-\mathrm{H}) .{ }^{13} \mathrm{CNMR}\left(125 \mathrm{MHz}, \mathrm{DMSO}-d_{6}\right): \delta 164.3$, $164.3,163.1,152.2,150.1,146.8,136.7,134.5,134.1,133.3,132.7,132.2,130.5,130.2,130.1,129.7,129.5,128.0$, 127.6, 127.3, 127.1, 126.6, 125.6, 118.3, 116.5, 112.4. HREI-MS: $m / z$ calcd for $\mathrm{C}_{26} \mathrm{H}_{16} \mathrm{~N}_{6} \mathrm{O}_{2}[\mathrm{M}]+444.1335$, Found 444.1322 .

4-(5-(isoquinolin-3-yl)-1,3,4-oxadiazol-2-yl)- $N^{\prime}$-(3,4,5-trimethoxybenzylidene)benzohydrazide (15). Yield: $55 \%,{ }^{1} \mathrm{H}-\mathrm{NMR}\left(500 \mathrm{MHz}, \mathrm{DMSO}-d_{6}\right) \delta 12.09(\mathrm{~s}, 1 \mathrm{H}, \mathrm{NH}), 9.5(\mathrm{~s}, 1 \mathrm{H}, \mathrm{Ar}-\mathrm{H}), 9.3(\mathrm{~s}, 1 \mathrm{H}$, Aldehydic- H), $8.8(\mathrm{~s}, 1 \mathrm{H}, \mathrm{Ar}-\mathrm{H}), 8.5(\mathrm{~m}, 4 \mathrm{H}, \mathrm{Ar}-\mathrm{H}), 8.2(\mathrm{~d}, J=6.7 \mathrm{~Hz}, 1 \mathrm{H}, \mathrm{Ar}-\mathrm{H}), 8.1(\mathrm{~m}, 2 \mathrm{H}, \mathrm{Ar}-\mathrm{H}), 7.9(\mathrm{t}$, $J=6.1 \mathrm{~Hz}, 1 \mathrm{H}, \mathrm{Ar}-\mathrm{H}) 7.8(\mathrm{t}, J=6.1 \mathrm{~Hz}, 1 \mathrm{H}, \mathrm{Ar}-\mathrm{H}), 7.2(\mathrm{~d}, J=6.5 \mathrm{~Hz}, 1 \mathrm{H}, \mathrm{Ar}-\mathrm{H}), 3.8(\mathrm{~s}, 9 \mathrm{H}, 3-\mathrm{OMe}){ }^{13} \mathrm{CNMR}$ $\left(125 \mathrm{MHz}\right.$, DMSO- $\left.d_{6}\right): \delta 164.3,164.3,163.1,153.4,153.2,152.2,150.1,146.5,141.3,136.4,132.5,130.3,130.1$, $130.0,129.3,128.3,128.1,127.6,127.4,127.2,126.8,125.4,116.3,104.0,104.0,60.5,55.9,55.9$. HREI-MS: $m / z$ calcd for $\mathrm{C}_{28} \mathrm{H}_{23} \mathrm{~N}_{5} \mathrm{O}_{5}[\mathrm{M}]+509.1699$, Found 509.1685.

$N^{\prime}$-(2-cyanobenzylidene)-4-(5-(isoquinolin-3-yl)-1,3,4-oxadiazol-2-yl)benzohydrazide (16). Yield: 68\%, ${ }^{1} \mathrm{H}-\mathrm{NMR}\left(500 \mathrm{MHz}, \mathrm{DMSO}-d_{6}\right) \delta 12.4(\mathrm{~s}, 1 \mathrm{H}, \mathrm{NH}), 9.5(\mathrm{~s}, 1 \mathrm{H}, \mathrm{Ar}-\mathrm{H}), 9.3(\mathrm{~s}, 1 \mathrm{H}$, AldehydicH), $8.9(\mathrm{~s}, 1 \mathrm{H}, \mathrm{Ar}-\mathrm{H}), 8.3(\mathrm{~m}, 4 \mathrm{H}, \mathrm{Ar}-\mathrm{H}), 8.2(\mathrm{~m}, 3 \mathrm{H}, \mathrm{Ar}-\mathrm{H}), 8.1(\mathrm{~d},(\mathrm{~d}, J=6.4 \mathrm{~Hz}, 1 \mathrm{H}, \mathrm{Ar}-\mathrm{H}), 7.10(\mathrm{~m}, 1 \mathrm{H}, \mathrm{Ar}-$ $\mathrm{H}), 7.9(\mathrm{t}, J=6.3 \mathrm{~Hz}, 1 \mathrm{H}, \mathrm{Ar}-\mathrm{H}), 7.8(\mathrm{t}, J=6.1 \mathrm{~Hz}, 1 \mathrm{H}, \mathrm{Ar}-\mathrm{H}), 7.6(\mathrm{~d}, J=5.3 \mathrm{~Hz}, 1 \mathrm{H}, \mathrm{Ar}-\mathrm{H}) .{ }^{13} \mathrm{CNMR}(125 \mathrm{MHz}$, DMSO- $d_{6}$ ): $\delta 164.3,164.3,163.1,152.2,150.1,143.1,136.5,134.3,132.6,132.2,131.5,133.1,130.1,130.0,129.9$, $129.6,129.3,128.2,127.5,127.3,127.2,126.7,125.7,116.6,115.6,111.7$. HREI-MS: $m / z$ calcd for $\mathrm{C}_{26} \mathrm{H}_{16} \mathrm{~N}_{6} \mathrm{O}_{2}$ $[\mathrm{M}]+444.1335$, Found 444.1322 .

$N^{\prime}$-(4-cyanobenzylidene)-4-(5-(isoquinolin-3-yl)-1,3,4-oxadiazol-2-yl)benzohydrazide (17). Yield: $73 \%,{ }^{1} \mathrm{H}-\mathrm{NMR}\left(500 \mathrm{MHz}\right.$, DMSO- $\left.d_{6}\right) \delta 12.3(\mathrm{~s}, 1 \mathrm{H}, \mathrm{NH}), 9.5(\mathrm{~s}, 1 \mathrm{H}, \mathrm{Ar}-\mathrm{H}), 8.8(\mathrm{~s}, 1 \mathrm{H}$, Aldehydic- $\mathrm{H}), 8.7$ (s, $1 \mathrm{H}, \mathrm{Ar}-\mathrm{H}), 8.3(\mathrm{~m}, 4 \mathrm{H}, \mathrm{Ar}-\mathrm{H}) 8.2(\mathrm{~m}, 4 \mathrm{H}, \mathrm{Ar}-\mathrm{H}), 8.1(\mathrm{~d}, J=6.7 \mathrm{~Hz}, 1 \mathrm{H}, \mathrm{Ar}-\mathrm{H}), 7.9(\mathrm{t}, J=6.2 \mathrm{~Hz}, 1 \mathrm{H}, \mathrm{Ar}-\mathrm{H}), 7.8(\mathrm{t}$, $J=6.8 \mathrm{~Hz}, 1 \mathrm{H}, \mathrm{Ar}-\mathrm{H}), 7.6$ (d, $J=6.8 \mathrm{~Hz}, 1 \mathrm{H}, \mathrm{Ar}-\mathrm{H}) .{ }^{13} \mathrm{CNMR}\left(125 \mathrm{MHz}\right.$, DMSO- $\left.d_{6}\right): \delta 164.3,164.3,163.1,152.2$, $150.1,146.5,138.0,136.5,132.4,132.1,132.0,129.5,130.5,130.2,130.0,128.0,127.7,127.5,127.3,126.9,126.3,126.1$, 125.6, 118.4, 116.5, 114.5. HREI-MS: $m / z$ calcd for $\mathrm{C}_{26} \mathrm{H}_{16} \mathrm{~N}_{6} \mathrm{O}_{2}[\mathrm{M}]+444.1335$, Found 444.1322.

$N^{\prime}$-(2-hydroxy-3-methoxybenzylidene)-4-(5-(isoquinolin-3-yl)-1,3,4-oxadiazol-2-yl)benzohydrazide (18). Yield: $73 \%,{ }^{1} \mathrm{H}-\mathrm{NMR}\left(500 \mathrm{MHz}, \mathrm{DMSO}-d_{6}\right): \delta 12.3(\mathrm{~s}, 1 \mathrm{H}, \mathrm{NH}), 9.5(\mathrm{~s}, 1 \mathrm{H}, \mathrm{Ar}-\mathrm{H}), 8.8(\mathrm{~s}, 1 \mathrm{H}$, Aldehydic- H), $8.7(\mathrm{~s}, 1 \mathrm{H}, \mathrm{Ar}-\mathrm{H}), 8.3(\mathrm{~m}, 4 \mathrm{H}, \mathrm{Ar}-\mathrm{H}), 8.2(\mathrm{~d}, J=6.4 \mathrm{~Hz}, 1 \mathrm{H}, \mathrm{Ar}-\mathrm{H}), 7.9(\mathrm{t}, J=6.1 \mathrm{~Hz}, 1 \mathrm{H}, \mathrm{Ar}-\mathrm{H})$, $7.8(\mathrm{t}, J=6 \mathrm{~Hz}, 1 \mathrm{H}, \mathrm{Ar}-\mathrm{H}), 7.2(\mathrm{~d}, J=6.2 \mathrm{~Hz}, 1 \mathrm{H}, \mathrm{Ar}-\mathrm{H}), 7.06(\mathrm{~d}, J=6.2 \mathrm{~Hz}, 1 \mathrm{H}, \mathrm{Ar}-\mathrm{H}), 6.9(\mathrm{~m}, 2 \mathrm{H}, \mathrm{Ar}-\mathrm{H}), 3.8$ (s, 3H, OMe). ${ }^{13} \mathrm{CNMR}\left(125 \mathrm{MHz}\right.$, DMSO- $\left.d_{6}\right): \delta 164.3,164.3,163.1,152.2,150.1,149.9,149.3,146.0,136.6,132.7$, 130.4, $130.4130 .1,130.0,129.3,128.1,127.7,127.1,126.6,125.5,124.2,119.3,116.5,116.4,115.0,55.9$. HREI-MS: $m / z$ calcd for $\mathrm{C}_{26} \mathrm{H}_{19} \mathrm{~N}_{5} \mathrm{O}_{4}[\mathrm{M}]+465.1437$, Found 465.1420 .

4-(5-(isoquinolin-3-yl)-1,3,4-oxadiazol-2-yl)- $N^{\prime}$-(2,3,4-trimethoxybenzylidene)benzohydrazide (19). Yield: 60\%, ${ }^{1} \mathrm{H}-\mathrm{NMR}\left(500 \mathrm{MHz}, \mathrm{DMSO}-d_{6}\right) \delta 12.0(\mathrm{~s}, 1 \mathrm{H}, \mathrm{NH}) 9.5(\mathrm{~s}, 1 \mathrm{H}, \mathrm{Ar}-\mathrm{H}) 8.8(\mathrm{~s}, 1 \mathrm{H}$, Aldehydic- H), $8.7(\mathrm{~s}, 1 \mathrm{H}, \mathrm{Ar}-\mathrm{H}), 8.3(\mathrm{~m}, 5 \mathrm{H}, \mathrm{Ar}-\mathrm{H}), 8.2(\mathrm{~d}, J=6.6 \mathrm{~Hz}, 1 \mathrm{H}, \mathrm{Ar}-\mathrm{H}), 7.9(\mathrm{t}, J=6.2 \mathrm{~Hz}, 1 \mathrm{H}, \mathrm{Ar}-\mathrm{H})$ $7.8(\mathrm{t}, J=6.2 \mathrm{~Hz}, 1 \mathrm{H}, \mathrm{Ar}-\mathrm{H}), 7.6(\mathrm{~d}, J=7.2 \mathrm{~Hz}, 1 \mathrm{H}, \mathrm{Ar}-\mathrm{H}) 6.9(\mathrm{~d}, J=7.4 \mathrm{~Hz}, 1 \mathrm{H}, \mathrm{Ar}-\mathrm{H}) 3.8(\mathrm{~s}, 9 \mathrm{H}, 3-\mathrm{OMe})$. 
${ }^{13} \mathrm{CNMR}\left(125 \mathrm{MHz}\right.$, DMSO- $\left.d_{6}\right): \delta 164.3,164.3163 .1,158.2,151.3,152.2,150.1,146.2,143.3,136.6,132.7,132.7$, $130.1,130.1129 .2,128.3,127.5,127.3,127.1,126.7,125.5,125.3,116.3,110.1,103.9,61.3,60.5,55.9$. HREI-MS: $\mathrm{m} / z$ calcd for $\mathrm{C}_{28} \mathrm{H}_{23} \mathrm{~N}_{5} \mathrm{O}_{5}[\mathrm{M}]+509.1699$, Found 509.1685.

4-(5-(isoquinolin-3-yl)-1,3,4-oxadiazol-2-yl)- $N^{\prime}$-(2,3,4-trihydroxybenzylidene)benzohydrazide (20). Yield: $54 \%,{ }^{1} \mathrm{H}-\mathrm{NMR}\left(500 \mathrm{MHz}, \mathrm{DMSO}-d_{6}\right) \delta 12.2(\mathrm{~s}, 1 \mathrm{H}, \mathrm{NH}) 9.5(\mathrm{~s}, 1 \mathrm{H}, \mathrm{Ar}-\mathrm{H}) 8.8$ (s, $1 \mathrm{H}$, Aldehydic$\mathrm{H}), 8.7(\mathrm{~s}, 1 \mathrm{H}, \mathrm{Ar}-\mathrm{H}), 8.3(\mathrm{~m}, 4 \mathrm{H}, \mathrm{Ar}-\mathrm{H}), 8.2(\mathrm{~d}, J=6.6 \mathrm{~Hz}, 1 \mathrm{H}, \mathrm{Ar}-\mathrm{H}), 7.9(\mathrm{t}, J=6.1 \mathrm{~Hz}, 1 \mathrm{H}, \mathrm{Ar}-\mathrm{H}), 7.8(\mathrm{t}$, $J=6.1 \mathrm{~Hz}, 1 \mathrm{H}, \mathrm{Ar}-\mathrm{H}), 7.6(\mathrm{~d}, J=7.4 \mathrm{~Hz}, 1 \mathrm{H}, \mathrm{Ar}-\mathrm{H}), 6.8(\mathrm{~d}, J=6.9 \mathrm{~Hz}, 1 \mathrm{H}, \mathrm{Ar}-\mathrm{H}), 8.4(\mathrm{~d}, J=6.9 \mathrm{~Hz}, 1 \mathrm{H}, \mathrm{Ar}-\mathrm{H})$. ${ }^{13} \mathrm{CNMR}\left(125 \mathrm{MHz}\right.$, DMSO- $\left.d_{6}\right): \delta 164.3,164.3,163.1,153.2,152.5,152.2,150.1,146.3,136.5,136.3,132.6,130.4$, $130.3,130.0,129.2,128.2,127.4,127.2,127.1,126.7,126.4,125.7,116.5,112.3,110.2$. HREI-MS: $m / z$ calcd for $\mathrm{C}_{25} \mathrm{H}_{17} \mathrm{~N}_{5} \mathrm{O}_{5}[\mathrm{M}]+467.1230$, Found 467.1213.

Assay protocol for thyimidine phosphorylase. The desired activity of thymidine phosphorylase under this protocol was carried out with slight modification in the method described by Krenitsky and Bush in 1979 ${ }^{46,47}$. Here, TP activity was determined through spectrophotometrically by absorbance at $290 \mathrm{~nm}$. Although the whole reaction mixture of $200 \mu \mathrm{L}$ have the limit of $145 \mu \mathrm{L}$ of potassium phosphate buffer ( $\mathrm{pH} 7.4,50 \mathrm{mM}$ ), $30 \mu \mathrm{L}$ of enzyme (human and E. coli) by concentration 0.05 and $0.002 \mathrm{U}$, distinectly, followed by incubation through $5 \mu \mathrm{L}$ of test materials $(0.075,0.15,0.3$ and $0.5 \mathrm{mM})$ at $25^{\circ} \mathrm{C}$ for $10 \mathrm{~min}$ in triplet reader. Subsequently, pre-read at $290 \mathrm{~nm}$ was taken to conclude the absorbance of concern substrate particles. The crave substrate "thyimidin" $(20 \mu \mathrm{L}, 1.5 \mathrm{mM})$, was then dissolved in the buffer (potassium phosphate), followed by instant addition to plate with constant read after every 10, 20 and $30 \mathrm{~min}$ in micro plate reader (spectra max, molecular devices, CA, USA). All the assay were implemented in triplicate under positive control of 7-Deazxanthine.

Molecular docking. The interactions between target and inhibitors were investigated through molecular docking ${ }^{48}$. Molecular docking was carried out through MOE-Dock program (www.chemcomp.com) to find the interactions between synthesized analogs and ligand proteins. From Protein Databank (PDB), three dimensional structures (3D) of thymidine phosphorylase (4EAD) was retrieved. In MOE (www.chemcomp.com) the synthesized analogs were docked into the active site of the target docked enzyme by applying the default parameter i-e Rescoring 1: London dG, Refinement: Forcefield (MMFF94x), Placement: Triangle Matcher, London dG: Rescoring 2. Ten conformations were generated for each in which the conformation of the top ranked based on docking score was selected for additional studies in molecular docking. The best pose here having polar, $\mathrm{Pi}-\mathrm{H}$ and $\mathrm{H}-\mathrm{Pi}$ interactions were analyzed by Pymol software after molecular docking.

Received: 30 May 2019; Accepted: 9 October 2019;

Published online: 05 November 2019

\section{References}

1. Siegel, R. et al. Cancer Statistics. CA: CA-Cancer. J. Clin. 63, 11-30 (2013).

2. Rowinsky, E. K. et al. The pursuit of optimal outcomes in cancer therapy in a new age of rationally designed target-based anticancer agents. Drugs. 60, 1-14 (2000)

3. Jarmula, A. Antifolate inhibitors of thymidylate synthase as anticancer drugs. Mini. Reviews. in Med. chem. 10, 1211-1222 (2010).

4. Bloom, J. D. et al. Evolving strategies for enzyme engineering. Current opinion in structural biology 15, 447-452 (2005).

5. Anna, K. et al. Novel purine nucleoside analogues forhematological malignancies. Recent patents on anti-cancer drug discovery. $\mathbf{3}$, 123-136 (2008)

6. Friedkin, M. et al. The enzymatic synthesis of nucleosides: I. Thymidine phosphorylase in mammalian tissue. J. Biol. Chem. 207, 245-256 (1954)

7. Krenitsky, T. A. et al. Specificity of mouse uridine phosphorylase. J. biol. Chem. 239, 805-812 (1964).

8. Schwartz, P. A. et al. Transition state analysis of the arsenolytic depyrimidination of thymidine by human thymidine phosphorylase. Biochemistry. 50, 1412-1420 (2011).

9. Matsushita, S. et al. The effect of a thymidine phosphorylase inhibitor on angiogenesis and apoptosis in tumors. Cancer. research. 59, 1911-1916 (1999).

10. Perez-Perez, M. J. et al. Thymidine phosphorylase inhibitors: recent developments and potential therapeutic applications. Mini. Rev. Med. Chem. 5, 1113-1123 (2005).

11. Moghaddam, A. et al. Thymidine phosphorylase is angiogenic and promotes tumor growth. Proc. Natl. Acad Sci. 92, 998-1002 (1995).

12. Takeuchi, M. et al. Aberrant production of gliostatin/platelet-derived endothelial cell growth factor in rheumatoid synovium. Arthritis-Rheumatism. 37, 662-672 (1994).

13. Creamer, D. et al. Overexpression of the angiogenic factor platelet-derived endothelial cell growth factor/thymidine phosphorylase in psoriatic epidermis. Brit. J. Dermatol. 137, 851-855 (1997).

14. Focher, F. et al. Thymidine phosphorylase: a two-face Janus in anticancer chemotherapy. Cur- Cancer. Drug. Targets. 1, 141-153 (2001).

15. Miyadera, K. et al. Novel functional antitumor nucleoside TAS-102, combined from of F3Rhd and its modulator (2): inhibitory effect of TPI on tumor-derived angiogenesis and metasi. Proc. Am. Assoc. Cancer. Res. 39, 609 (1998).

16. Pomeisl, K. et al. Syntheses of pyrimidine acyclic nucleoside phosphonates as potent inhibitors of thymidine phosphorylase (PDECGF) from SD-lymphoma. Nucleosides, Nucleotides. and Nucleic Acids. 26, 1025-1028 (2007).

17. Abdul, G. et al. Thymidine phosphorylase from Escherichia coli: Tight-binding inhibitors as enzyme active-site titrants. J. Enzyme. Inhib. Med. Chem. 21, 69-73 (2006).

18. Nencka, R. et al. Discovery of 5-substituted-6-chlorouracils as efficient inhibitors of human thymidine phosphorylase. Med. Chem. 50, 6016-6023 (2007)

19. Sohail, A. S. et al. Synthesis of pyrazolo[1,5-a] $[1,3,5]$ triazine derivatives as inhibitors of thymidine phosphorylase. Eur. J. Med. Chem. 65, 1-11 (2013)

20. Liekens, S. et al. Nucleosides, Nucleotides. Nucleic Acids. 25, 975-975 (2006).

21. Casanova, E. et al. 5 '-O-Tritylinosine and Analogues as Allosteric Inhibitors of Human Thymidine Phosphorylase. J. Med Chem. 49, 5562-5570 (2006). 
22. Pyo, M. K. et al. Effects of higenamine and its 1-naphthyl analogs, YS-49 and YS-51, on platelet TXA2 synthesis and aggregation. Thrombosis. research. 120, 81-86 (2007).

23. Olga, L. M. et al. Derivatives of 7-amino-1, 2,3,4-tetrahydroisoquinoline and isophthalic acids as novel fibrinogen receptor antagonists. Bioorg. Med. Chem. Lett. 16, 5294-5297 (2006).

24. Lei, B. Global, local and novel consensus quantitative structure-activity relationship studies of 4-(phenylaminomethylene) isoquinoline-1, 3 (2H, 4H)-diones as potent inhibitors of the cyclin-dependent kinase. Anal. Chimica.acta. 644, 17-24 (2009).

25. Ping, C. et al. Identification of Novel and Potent Isoquinoline. Bioorg. Med. Chem. Lett. 13, 1345-1348 (2003).

26. Anand, R. et al. Toward the Development of a Potent and Selective Organoruthenium Mammalian Sterile 20 Kinase Inhibitor. J. Med. Chem. 52, 1602-1611 (2009).

27. Morrell, A. et al. Investigation of the lactam side chain length necessary for optimal indenoisoquinoline topoisomerase I inhibition and cytotoxicity in human cancer cell cultures. J. Med. Chem. 50, 2040-2048 (2007).

28. Gollapalli, M. et al. Synthesis of Bis-indolylmethane Sulfonohydrazides derivatives as potent $\alpha$-Glucosidase inhibitors. Bioorg. Chem. 80, 112-120 (2018).

29. Noreen, T. et al. Synthesis of alpha amylase inhibitors based on privileged indole scaffold. Bioorg. Chem. 72, 248-255 (2017).

30. Rahim, F. et al. Development of bis-Thiobarbiturates as Successful Urease Inhibitors and their Molecular Modeling Studies. Chin. Chem. Lett. 27, 693-697 (2016).

31. Taha, M. et al. Synthesis and biological evaluation of novel $\mathrm{N}$-arylidenequinoline-3-carbohydrazides as potent $\beta$-glucuronidase inhibitors. Bioorg. Med. Chem. 24, 3696-3704 (2016).

32. Rashid, U. et al. Synthesis of 2-acylated and sulfonated 4-hydroxycoumarins: In vitro urease inhibition and molecular docking studies. Bioorg. Chem. 66, 111-116 (2016).

33. Rahim, F. et al. Triazinoindole analogs as potent inhibitors of $\alpha$-glucosidase: Synthesis, biological evaluation and molecular docking studies. Bioorg. Chem. 58, 81-87 (2015).

34. Taha, M. et al. Synthesis, $\beta$-Glucuronidase Inhibition and Molecular Docking Studies of Hybrid Bisindole-Thiosemicarbazides Analogs. Bioorg. Chem. 68, 56-63 (2016).

35. Taha, M. et al. Biology-oriented drug synthesis (BIODS) of 2-(2-methyl-5-nitro-1Himidazol-1-yl)ethyl aryl ether derivatives, in vitro $\alpha$-amylase inhibitory activity and in silico studies. Bioorg. Chem. 74, 1-9 (2017).

36. Rahim, F. et al. Synthesis, in vitro evaluation and molecular docking studies of thiazole derivatives as new inhibitors of $\alpha$-glucosidase. Bioorg. Chem. 62, 15-21 (2015).

37. Rahim, F. et al. Synthesis, Molecular Docking, Acetylcholinesterase and Butyrylcholinesterase Inhibitory Potential of Thiazole Analogs as New Inhibitors for Alzheimer Disease. Bioorg. Chem. 62, 106-116 (2015).

38. Rahim, F. et al. Isatin based Schiff bases as inhibitors of $\alpha$-glucosidase: Synthesis, characterization, in vitro evaluation and molecular docking studies. Bioorg. Chem. 60, 42-48 (2015).

39. Rahim, F. et al. Synthesis and in vitro Acetylcholinesterase and Butyrylcholinesterase Inhibitory Potential of Hydrazide based Schiff Bases. Bioorg. Chem. 68, 30-40 (2016).

40. Taha, M. et al. Bisindolylmethane thiosemicarbazides as potential inhibitors of urease: Synthesis and molecular modeling studies. Bioorg. Med. Chem. 26, 152-160 (2018).

41. Taha, M. et al. Synthesis of bis-indolylmethanes as new potential inhibitors of $\beta$-glucuronidase and their molecular docking studies. Eur. J. Med. Chem. 143, 1757-1767 (2018).

42. Almandil, N. B. et al. Synthesis of Thymidine Phosphorylase InhibitorBased on Quinoxaline Derivatives and TheirMolecular Docking Study. Molecules. 24, 1002 (2019).

43. Uddin, I. et al. Synthesis and molecular docking study of piperazine derivatives as potent inhibitor of thymidine phosphorylase. Bioorg. Chem. 78, 324-331 (2018).

44. Taha, M. et al. Synthesis, molecular docking study and thymidine phosphorylase inhibitory activity of 3-formylcoumarin derivatives. Bioorg. Chem 78, 17-23 (2018).

45. Ullah, H. et al. Molecular docking study and in vitro Thymidine Phosphorylase Inhibitory Potential of Oxadiazole Derivatives. Bioorg. Chem. 78, 58-67 (2018).

46. Krenitsky, T. A. et al. Bush by SRM Enzymatic assay of thymidine phosphorylase (EC 2.4. 2.4). US. Pat. 212.1 (1979).

47. Taha, M. et al. In silico binding analysis and SAR elucidations of newly designed benzopyrazine analogs as potent inhibitors of thymidine phosphorylase. Bioorg. Chem. 68, 80-89 (2016).

48. Andrew, A. L. et al. Prediction of Protein-Ligand Interactions. Docking and Scoring: Successes and Gaps. J. Med. Chem. 49, 5851-5855 (2006).

\section{Acknowledgements}

The authors are thankful to Higher Education Commission (HEC) Pakistan for support through Project No. 5721 \& 5092 under the National Research Program for Universities.

\section{Author contributions}

Conceptualization, M.T. and K.Z..; methodology, M.T.; software, A.W.; validation, F.R., K.Z. and H.U.; formal analysis, F.R.; investigation, S.A.A.S.; resources, M.T.; data curation, Q.U.A. and S.A.A.S.; Writing-Original Draft preparation, M.T. and Z.A.Z.; Writing-Review and Editing, F.R. and Z.A.Z.; visualization, S.A.A.S.; supervision, F.R.; project administration, Z.A.Z. and M.T.; funding acquisition, M.T. and Q.U.A.

\section{Competing interests}

The authors declare no competing interests.

\section{Additional information}

Supplementary information is available for this paper at https://doi.org/10.1038/s41598-019-52100-0.

Correspondence and requests for materials should be addressed to M.T. or Z.A.Z.

Reprints and permissions information is available at www.nature.com/reprints.

Publisher's note Springer Nature remains neutral with regard to jurisdictional claims in published maps and institutional affiliations. 
(c) (i) Open Access This article is licensed under a Creative Commons Attribution 4.0 International License, which permits use, sharing, adaptation, distribution and reproduction in any medium or format, as long as you give appropriate credit to the original author(s) and the source, provide a link to the Creative Commons license, and indicate if changes were made. The images or other third party material in this article are included in the article's Creative Commons license, unless indicated otherwise in a credit line to the material. If material is not included in the article's Creative Commons license and your intended use is not permitted by statutory regulation or exceeds the permitted use, you will need to obtain permission directly from the copyright holder. To view a copy of this license, visit http://creativecommons.org/licenses/by/4.0/.

(C) The Author(s) 2019 